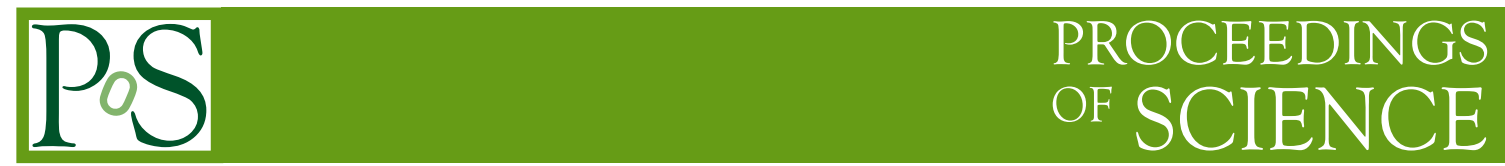

\title{
Automated Lattice Perturbation Theory
}

\author{
Christopher Monahan* \\ College of William and Mary \\ E-mail: c jmonahan@wm.edu
}

I review recent developments in automated lattice perturbation theory. Starting with an overview of lattice perturbation theory, I focus on the three automation packages currently "on the market": HiPPy/HPsrc, Pastor and PhySyCAl. I highlight some recent applications of these methods, particularly in $B$ physics. In the final section I briefly discuss the related, but distinct, approach of numerical stochastic perturbation theory.

31st International Symposium on Lattice Field Theory - LATTICE 2013

July 29 - August 3, 2013

Mainz, Germany

${ }^{*}$ Speaker. 


\section{Introduction}

The lattice is generally regarded as a nonperturbative tool. This does not mean, however, that perturbative analyses of lattice field theories are redundant. Many of the early calculations in lattice field theory incorporated analytic treatments of weak coupling behaviour, because initial efforts were often constrained by insufficient computational power. As lattice field theory matured, increasing computing power and improved algorithms facilitated fully nonperturbative treatments with greater precision. Parallel theoretical developments, such as the clarification of conceptual issues with the pole mass, often demonstrated the need for fully nonperturbative treatments, particularly when aiming for high precision

Nevertheless, lattice perturbation theory, which is perhaps better thought of as "perturbation theory for lattice actions", has retained an important role for a variety of lattice calculation. Many of these applications fall into one of three general categories (see, for example, the references collected in [1]): determining the renormalization parameters of bare lattice actions; matching regularisation schemes and extracting continuum results from lattice data; and improving lattice actions. These categories are inter-related and can be broadly construed as accounting for the effects of energy excluded by the lattice cutoff. In other words, LPT calculations are generally concerned with renormalisation.

In the next section I discuss the motivation and justification for LPT and in Section 3 I review automated lattice perturbation theory, focussing on the three automation packages currently available and highlighting some applications of these techniques. In Section 4 I discuss the related, but distinct, approach of numerical stochastic perturbation theory.

\section{Perturbative and nonperturbative renormalisation}

The lattice is a gauge-invariant ultraviolet regulator that, by discretising spacetime, excludes all momenta greater than $\pi / a$ (where $a$ is the lattice spacing). To correctly account for energy scales eliminated by the cutoff, the regularised theory must be renormalised. In continuum gauge theories, one is generally restricted to perturbative renormalisation, but on the lattice both perturbative and nonperturbative techniques are available.

There is a wide variety of nonperturbative methods and a full discussion is beyond the scope of these proceedings, but a partial list includes nonperturbative tuning via physical quantities or dispersion relations (see, for example, [2]); imposing Ward identities or chiral symmetry relations [3]; and step-scaling methods, which have been applied to the Schrödinger functional [4], off-shell Green functions (the "Rome-Southampton method") [5], physical quantities [6] and gradient flow smearing procedures [7].

Although different in practice, nonperturbative methods share two distinct advantages. The first is that nonperturbative techniques eliminate perturbative truncation errors, which can be hard to quantify, in favour of statistical and systematic errors, which can generally be reliably determined and, with intensified computational effort, systematically reduced. The second is more aesthetic: only a fully nonperturbative approach is truly ab initio. 
Unfortunately there are disadvantages: step-scaling and iterative methods incur a greatly increased computational expense, matching to physical quantities results in a loss of predictive power and some methods explicitly break gauge invariance.

Lattice perturbation theory (LPT) is often an attractive alternative, computationally cheaper and without loss of predictive power. Naturally there is a cost. LPT introduces perturbative truncation errors and as lattice calculations become more precise, the perturbative errors often become the largest source of uncertainty in the final result. Furthermore, reliably estimating higher-order truncation errors can be difficult. In many cases, though, the use of LPT is justified by a consideration of the relevant energy scales in question. For current lattice calculations the scales excluded by the inverse lattice spacing are those above (approximately) $5 \mathrm{GeV}$. At such scales, the coupling constant is sufficiently small, $\alpha_{s}(\pi / a) \sim 0.2$, that perturbative approximations to the renormalisation parameters are likely to be valid.

LPT therefore connects the low and high energy regimes of a lattice field theory. For lattice quantum chromodynamics (QCD) this connection has been tested and validated in a wide range of processes by comparing perturbative calculations with polynomial fits (in the coupling constant) of nonperturbative data in the weak coupling regime [8-13].

This isn't quite the whole story, however. Early LPT calculations were plagued by slow convergence and inconsistent results [14]. These issues were the consequence of a poor choice of expansion parameter: the bare lattice coupling $\alpha_{\text {latt }}=g_{0}^{2} /(4 \pi)$. The convergence of the perturbative series can be improved by introducing a different coupling constant [14]. For example, one common choice of scheme for lattice QCD is the "V-scheme" [15], expressed at an appropriately chosen scale, usually the "BLM scale" $[15,16]$, that characterises the scale of the process concerned. The convergence of the perturbative expansion can be enhanced by tadpole improvement [14], which partially mitigates the effects of high momenta contributions from so-called tadpole diagrams. This improvement has now been generalised to remove "cactus" diagrams [17], although this technique is less widely used.

The main challenge for perturbative renormalisation is the complexity of loop calculations in LPT. Even for the simplest lattice actions, the use of gauge links rather than gauge fields increases the number of Feynman diagrams that must be evaluated at a given order by generating vertices with an arbitrary number of gauge fields (consistent with the order of the calculation, of course).

For many modern lattice actions, however, things are worse. Sophisticated lattice actions often include irrelevant operators, smeared gauge links to improve discretisation effects, or tadpole (or cactus) improvement, and these considerably complicate the Feynman rules. Highly improved actions, such as the highly-improved staggered quark (HISQ) and nonrelativistic QCD (NRQCD) actions, generate Feynman rules that cannot feasibly be manipulated by hand. Furthermore, the lack of Lorentz symmetry ensures that Feynman integrands are no longer amenable to the same tricks and transformations that are available for continuum theories. To cope with all of these complications LPT has been automated.

Before proceeding, it is worth considering what "automation" involves in the context of LPT. A formal definition is not necessary, but to convey a general sense of what I mean by "automation" I represent a paradigm example of an ALPT algorithm in Figure 1. The extent to which the user must fill in the steps represented by the "black box" at the heart of the process provides a heuristic measure of the level of automation of a particular routine. 


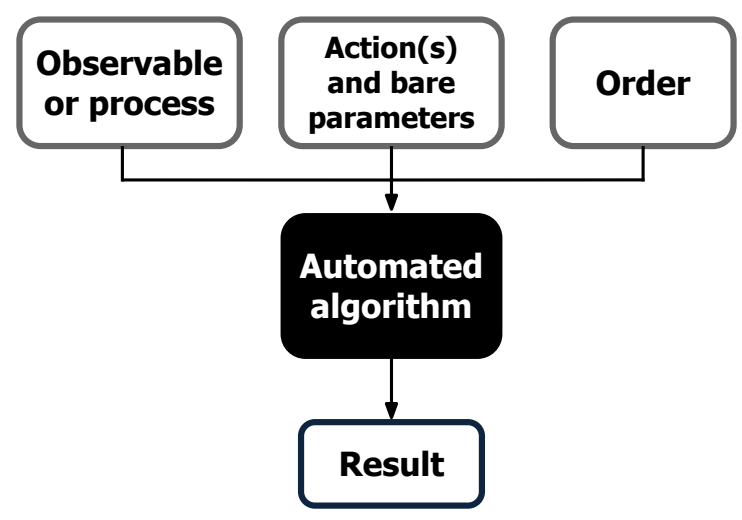

Figure 1: Flowchart illustrating an idealised, "black box" automation process.

\section{Automated routines}

The dawn of ALPT dates to the 1980s, when Lüscher and Weisz first proposed an algorithm for the automation of perturbative calculation in lattice gauge theories [18]. Computer algebra tools for analytic calculations in LPT were developed in [19] and [20], but it was not until twenty years after Lüscher and Weisz's proposal that full automation started to receive greater attention within the lattice community. Hart et al. extended the Lüscher-Weisz algorithm to fermions in [21] and similar ideas were independently developed for the Schrödinger functional [22]. In the last three years two new ALPT routines have been developed, Pastor [23] and PhySyCAl [24], and these, in conjunction with HiPPy/HPsrc, will be the focus of this section.

\subsection{HiPPy/HPsrc}

HiPPy and HPSIC are a suite of routines that automate perturbative calculations for lattice QCD. HiPPy is an implementation of the Lüscher-Weisz algorithm in Python, extended to include the fermion sector. HiPPy encodes the resulting Feynman rules as "vertex files", which are read into HPSIC, a set of FORTRAN modules that evaluate the relevant Feynman diagrams numerically.

This process is not fully automated, in the sense of Figure 1, in that the user must construct the desired Feynman diagrams by hand, but this procedure is conceptually identical to the standard application of the Feynman rules in continuum QCD. Modules within HP src define functions that correspond to each vertex and propagator, including fermions, ghosts and gauge vertices up to fivegluon interactions. Feynman diagrams are constructed from these functions in the usual manner and then evaluated numerically via VEGAS [25] or exact mode summation (for finite volume lattices).

The Lüscher-Weisz algorithm in HiPPy/HPsrc To illustrate in more detail how the process of deriving Feynman rules may be automated, I will briefly outline the Lüscher-Weisz algorithm, as implemented in HiPPy, and restrict the discussion to the gauge sector for brevity. The extension to fermions is fairly straightforward and documented in [21].

At weak coupling we may represent the link variables as exponentials of the gauge fields. In general the gauge action is built from gauge invariant, i.e. closed, loops of link variables that can 
be represented in momentum space as

$$
L(x, y ; U)=\sum_{r} \frac{(a g)^{r}}{r !} \sum_{k_{1}, \mu_{1}, a_{1}} \ldots \sum_{k_{r}, \mu_{r}, a_{r}} \widetilde{A}_{\mu_{1}}^{a_{1}}\left(k_{1}\right) \ldots \widetilde{A}_{\mu_{r}}^{a_{r}}\left(k_{r}\right) \times V_{r}\left(k_{1}, \mu_{1}, a_{1} ; \ldots ; k_{r}, \mu_{r}, a_{r}\right),
$$

where the $V_{r}$ are referred to as "vertex functions". These vertex functions can be further decomposed into a colour matrix, $C_{r}$, and a "reduced vertex", $Y_{r}$, that depends only on the momenta and positions of the links. This reduced vertex can be written as a product of exponentials

$$
V_{r}\left(k_{i}, \mu_{i}, a_{i}\right)=C_{r}\left(a_{i}\right) Y_{r}\left(k_{i}, \mu_{i}\right)=C_{r}\left(a_{i}\right) \sum_{n=1}^{n_{r}} f_{n} \exp \left[\frac{i}{2}\left(k_{1} \cdot v_{1}^{(n)}+\ldots+k_{r} \cdot v_{r}^{(n)}\right)\right],
$$

where $i \in\{1, \ldots, r\}$. Here the amplitudes associated with each term are denoted $f_{n}$ and the $v^{(n)}$ are the locations of each of the $r$ factors of the gauge potential. Therefore, for each combination of $r$ Lorentz vertices, there are $n_{r}$ terms, each with a corresponding amplitude $f_{n}$ and location $v_{r}^{(n)}$. Written in this form, the reduced vertices are encoded in ordered lists of "entities", $E=$ $\left(\mu_{1}, \ldots, \mu_{r} ; x, y ; v_{1}, \ldots, v_{r} ; f\right)$, which are stored in the vertex file generated by HiPPy.

The Feynman rules, each written to a separate vertex file, are constructed by repeatedly applying the convolution formulae derived in [26]. So, for example, for an action of the form $\mathscr{O}_{A} \mathscr{O}_{B}$, the Feynman rule for, say, the three-gluon vertex is the convolution of the Feynman rules for three gluons chosen from operator $\mathscr{O}_{A}$ with no gluons from $\mathscr{O}_{B}$, plus the corresponding convolution for two gluons from $\mathscr{O}_{A}$ with one from $\mathscr{O}_{B}$ and so on.

The colour structure is completely encoded in the matrices $C_{r}$. For the HiPPy/HPs sc package, this is handled in the HPsrc routines when the Feynman diagrams are constructed. Derivatives are obtained by algebraic manipulation of the reduced vertices, which ensures that no numerical derivatives are required and that the same vertex files can be used for both periodic and twisted boundary conditions. Within HPsrC, analytic derivatives are implemented using the derived taylor type, defined as part of the TaylUR package for FORTRAN [27].

\subsection{Two recent calculations with HiPPy/HPsrc}

A number of calculations have now been carried out using HiPPy/HPsrc (see, for example, [28-30]) and the code has been extensively tested. I would like to highlight two recent examples. For other applications, please see the review article [30].

Background field gauge in HiPPy/HPsrc The first of these developments is the implementation of background field gauge in HiPPy/HP src. Background field gauge (BFG) is a well established tool for gauge theories that has a number of advantages for calculations involving effective theories such as NRQCD.

Current lattice spacings are too coarse to directly incorporate $b$ quarks at their physical mass (whilst keeping lattice volumes large enough to avoid sizable finite volume effects). Traditionally this problem has been tackled by using an effective theory such as NRQCD or heavy quark effective theory (HQET) or with a relativistic heavy quark (RHQ) action that interpolates smoothly between relativistic and nonrelativistic limits. More recently, relativistic calculations that are extrapolated up to the physical $b$ mass have been developed [32]. Despite many advances, going to finer lattices is computationally very expensive and it is beneficial to retain an array of approaches to a problem 
to help identify and eliminate sources of systematic bias in lattice results. NRQCD and HQET remain an important ingredient in heavy quark physics on the lattice.

As part of the HPQCD Collaboration's programme of precision heavy quark physics, ALPT has been used to calculate the improvement coefficients for highly-improved NRQCD. The NRQCD action, which may be written as

$$
S=\sum_{x, t} \Psi^{\dagger}(x, t)\left[\Psi(x, t)-\left(1-\frac{a \delta H}{2}\right)\left(1-\frac{a H_{0}}{2 n}\right)^{n} U_{4}^{\dagger}\left(1-\frac{a H_{0}}{2 n}\right)^{n}\left(1-\frac{a \delta H}{2}\right) \Psi(x, t-a)\right],
$$

includes both the leading nonrelativistic kinetic energy term, $H_{0}=\nabla^{2} /\left(2 a m_{Q}\right)$ and corrections:

$$
\begin{aligned}
a \delta H= & -c_{1} \frac{\left(\Delta^{(2)}\right)^{2}}{8\left(a m_{Q}\right)^{3}}+c_{2} \frac{i g}{8\left(a m_{Q}\right)^{2}}(\nabla \cdot \widetilde{E}-\widetilde{E} \cdot \nabla)-c_{3} \frac{g}{8\left(a m_{Q}\right)^{2}} \sigma \cdot(\widetilde{\nabla} \times \widetilde{E}-\widetilde{E} \times \widetilde{\nabla}) \\
& -c_{4} \frac{g}{2 a m_{Q}} \sigma \cdot \widetilde{B}+c_{5} \frac{a^{2} \Delta^{(4)}}{24 a m_{Q}}-c_{6} \frac{a\left(\Delta^{(2)}\right)^{2}}{16 n\left(a m_{Q}\right)^{2}} .
\end{aligned}
$$

The coefficients of these corrections are normalised to unity at tree-level, but radiative corrections alter their value. These radiative corrections can be determined at one-loop using ALPT and the first results, for the leading relativistic and discretisation corrections $\left(c_{1}, c_{5}\right.$ and $\left.c_{6}\right)$, were presented in [29].

More recently, the one-loop radiative corrections to the chromo-magnetic, $c_{4}$, and Darwin, $c_{2}$, terms were calculated using BFG [31]. Using BFG greatly simplifies the matching calculation by

- ensuring that only gauge-covariant $D>4$ operators appear in the NRQCD effective action;

- rendering the relevant one-particle irreducible vertex function ultraviolet finite; and

- constraining renormalisation parameters via an extra background field Ward identity.

The first of these guarantees that we need only include gauge-invariant operators in the action and the second allows us to match lattice NRQCD directly to QCD. The third simplifies the calculation by reducing the number of renormalisation parameters that must be calculated.

The effectiveness of this improvement programme is demonstrated by the improvement in the lattice NRQCD result for the bottomonium hyperfine splitting, $\left(M_{\Upsilon}(1 S)-M_{\eta_{b}}(1 S)\right)$, when radiative corrections are included in the NRQCD action [33]. An earlier determination by the HPQCD Collaboration obtained 56(2) MeV for the hyperfine splitting, in disagreement with the world experimental average of 62.2(3.2) MeV [34]. Incorporating radiative corrections (amongst other improvements), HPQCD Collaboration's new result is $62.8(6.7) \mathrm{MeV}$, now in good agreement with experimental data.

The $b$ quark mass from ALPT and weak-coupling computations The HPQCD Collaboration's new determination of the $b$ quark mass from lattice NRQCD incorporated a mixed approach to determine perturbative results to next-to-leading order (i.e. two-loops).

Quark masses are conventionally expressed in the $\overline{M S}$ scheme, and for the $b$ quark, typically evaluated at a scale equal to the $b$ quark mass itself, $\bar{m}_{b}\left(\bar{m}_{b}\right)$. The $\overline{M S}$ scheme is inherently perturbative and the $\overline{M S}$ mass must be related to nonperturbative quantities (determined on the lattice) 
via a two stage matching procedure with the pole mass as an intermediate step [35]. For NRQCD, one method is to use the heavy quark energy shift, $E_{0}$,

$$
\bar{m}_{b}(\mu)=\frac{1}{2} Z_{M}^{-1}(\mu)\left[M_{\Upsilon}^{\text {expt }}-a^{-1}\left(a E_{\Upsilon}^{\text {sim }}-2 a E_{0}\right)\right] .
$$

Here $Z_{M}$ is the renormalisation parameter relating the pole-mass to the $\overline{M S}$ mass, known to threeloops [36], $M_{\Upsilon}^{\text {expt }}$ is the experimental $\Upsilon$ meson mass (corrected for missing electromagnetic effects) and $E_{\Upsilon}^{\text {sim }}$ is the ground state energy of the $\Upsilon$ meson determined from lattice NRQCD. The quantity $\left(a E_{\Upsilon}^{\text {sim }}-2 a E_{0}\right)$ represents the "binding" energy of the heavy quarks.

The HPQCD Collaboration's earlier result from NRQCD, $\bar{m}_{b}\left(\bar{m}_{b}\right)=4.4(3) \mathrm{GeV}$, was dominated by the two-loop perturbative uncertainty in the energy shift, $E_{0}$ [37]. By including not only the two-loop contribution to the energy shift, but also the three-loop quenched contribution as well, the error was reduced to the one percent level: $\bar{m}_{b}\left(\bar{m}_{b}\right)=4.166(43) \mathrm{GeV}$ [35].

In this calculation, the two-loop quenched contributions to the energy shift, which include all 27 gauge and ghost field diagrams and propagator insertions, were determined from weak coupling computations of lattice NRQCD in the quenched approximation. Results were obtained for a range of bare coupling values and the perturbative coefficients extracted from a simultaneous fit to a polynomial in the strong coupling constant and the inverse lattice size. The remaining fermionic diagrams were calculated using ALPT.

This approach combined the advantage of the (relative) computational speed of quenched lattive calculations with the (relative) simplicity of calculating only four diagrams with ALPT. The decrease in overall uncertainty in the final result for the $b$ quark mass is largely due to the inclusion of the two-loop (and quenched three-loop) contributions to the heavy quark energy shift. Such improvement demonstrates the effectiveness of the mixed approach to higher-order perturbation theory and underscores the importance of ALPT for precision lattice calculations.

\subsection{Pastor}

The ALPT package Pastor is a suite of routines based on the Lüscher-Weisz algorithm and tailored to the Schrödinger functional (SF) scheme. Developed by Dirk Hesse, the details of the implementation are discussed in [38] and initial calculations outlined in [23].

I illustrate the structure of Pastor in Figure 2. Vertices are generated by libsculptr, the $\mathrm{C}++$ backend of Pastor. Feynman diagrams are generated and evaluated by a set of Python routines: parse.py, run.py, and analysis.py. The user specifies all inputs (action, parameters, observable etc.) in an xml file, which is parsed by parse.py that, in turn, generates efficient $\mathrm{C}++$ code to evaluate the relevant diagrams. This $\mathrm{C}++$ code is called by run . $\mathrm{py}$ and written in a format that is easy to parse with the analysis script analysis.py. Feynman diagrams can be automatically created using the feynmf package in $\mathrm{LTT}_{\mathrm{E}} \mathrm{X}$.

\subsection{Matching HQET and QCD with Pastor}

Lattice HQET is an approach for heavy quarks on the lattice that is suitable for heavy-light mesons (for an overview, see, for example, [39]) and is currently being used by, among others, the ALPHA Collaboration. One of the principle differences from NRQCD is that higher order terms 


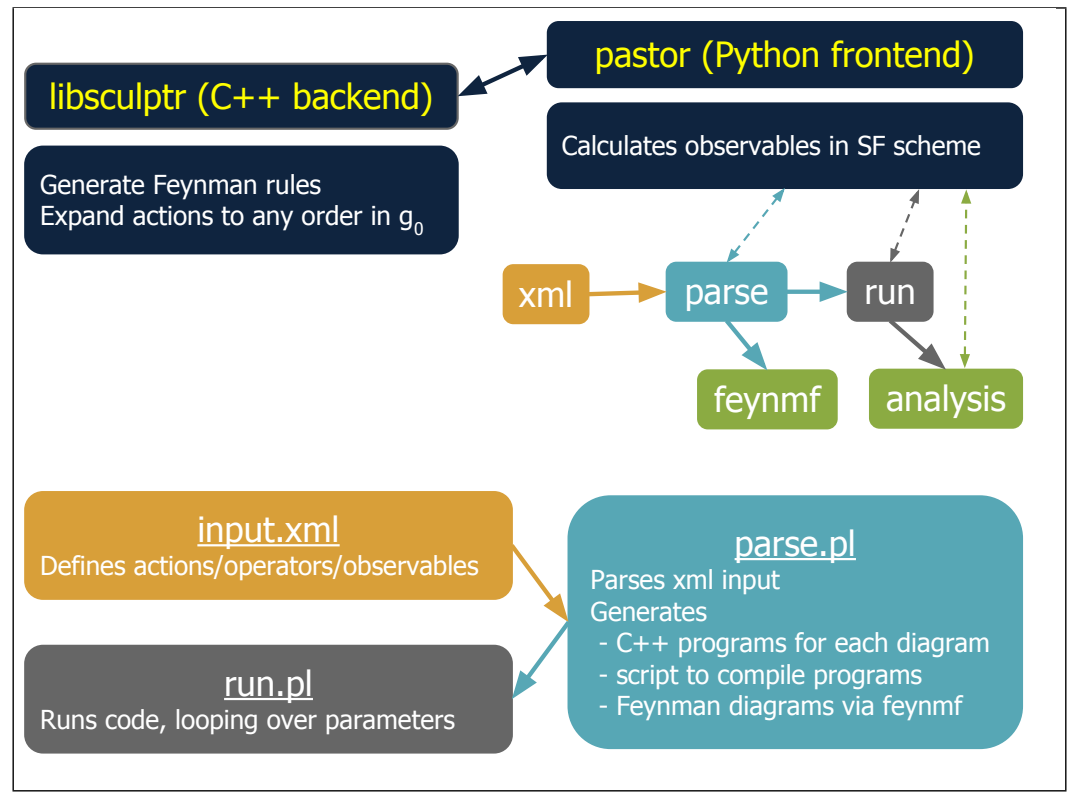

Figure 2: Schematic representation of the structure of Pastor. See accompanying text for details.

are included as operator insertions, rather than directly in the action. This ensures that HQET is renormalisable. The HQET Lagrangian at $\mathscr{O}\left(1 / m_{Q}\right)$ may be written as

$$
\mathscr{L}_{\text {HQET }}=\mathscr{L}_{\text {stat }}-\left(\omega_{\text {kin }} \mathscr{L}_{\text {kin }}+\omega_{\text {spin }} \mathscr{L}_{\text {spin }}\right)+\mathscr{O}\left(1 / m_{Q}^{2}\right),
$$

where $\mathscr{L}_{\text {stat }}$ is the leading order static (i.e. infinite mass limit) Lagrangian and $\mathscr{L}_{\text {kin }}$ and $\mathscr{L}_{\text {spin }}$ are the relativistic and chromo-magnetic corrections respectively. Correlation functions of operators are given by

$$
\langle O\rangle_{\mathrm{HQET}}=\langle O\rangle_{\mathrm{stat}}+\omega_{\text {kin }} \sum_{x}\left\langle O \mathscr{L}_{\text {kin }}(x)\right\rangle_{\text {stat }}+\omega_{\text {spin }} \sum_{x}\left\langle O \mathscr{L}_{\text {spin }}(x)\right\rangle_{\text {stat }}
$$

Lattice HQET must be matched to continuum QCD to extract physically meaningful results and this matching must be nonperturbative to avoid renormalon ambiguities. Lattice HQET observables are matched to continuum QCD via

$$
\phi_{i}^{\mathrm{QCD}}(L, z, a=0)=\phi_{i}^{\mathrm{HQET}}(L, z, a ; \omega(z, a))=\phi_{i}^{\mathrm{stat}}(L, a)+\phi_{i j}^{(1 / z)}(L, a) \omega_{j}(z, a) .
$$

Here $L$ is the lattice size, $a$ the lattice spacing, and $z$ is the dimensionless mass $z=\bar{m}(L) L$.

Achieving the one percent precision necessary for precision $B$ physics requires matching at $\mathscr{O}\left(1 / m_{Q}\right)$. At this order, there are nineteen parameters that must be fixed. Computationally, this is a very expensive task and to guide the process ALPT was used to investigate the $\mathscr{O}\left(1 / \mathrm{m}_{Q}^{2}\right)$ dependence of different operators and test the quality of observables for matching [23, 40].

The mass dependence can be studied by examining the ratio

$$
R_{\phi_{i}}=\frac{\phi_{i}^{\mathrm{QCD},(1)}(z)-\phi_{i}^{\mathrm{stat},(1)}}{\phi_{i}^{\mathrm{QCD},(0)}(z)-\phi_{i}^{\mathrm{stat},(0)}}
$$



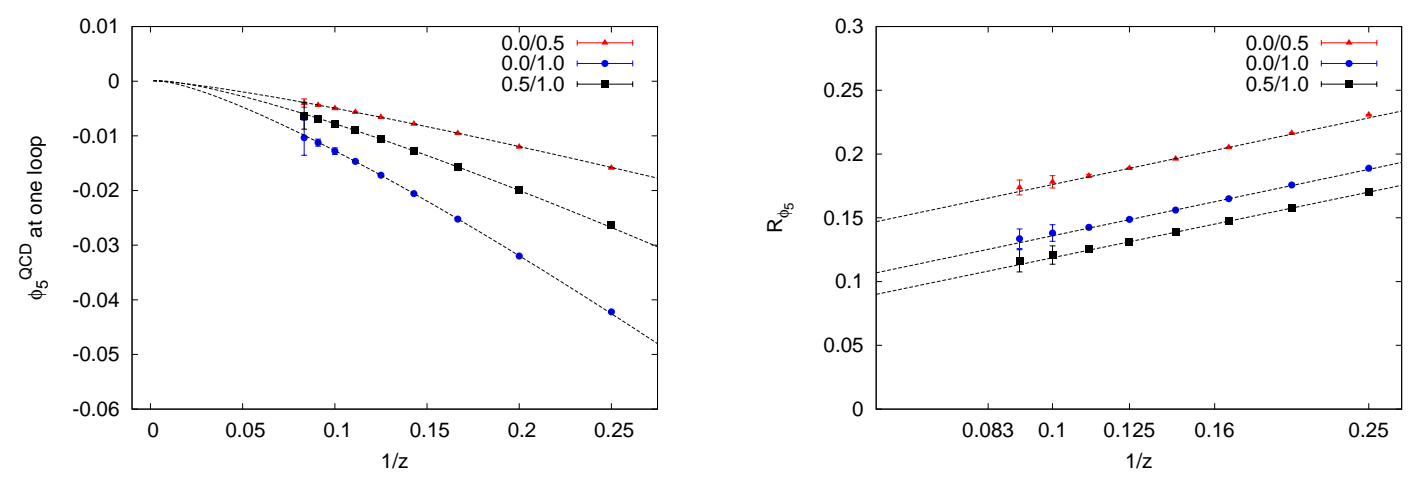

Figure 3: Mass dependence of the HQET observable $\phi_{5}$. For more details see the accommpanying text and Ref. [40].

and then plotting the result on a linear-log plot. Deviations from linear behaviour indicate $1 / z^{2}$ contributions and the slope gives the coefficient of subleading logarithmic behaviour. An example of such a plot is given in Figure 3, which shows the mass dependence of the observable $\phi_{5}$ (for details and definitions, see [40]). The righthand plot demonstrates that there is little contamination by $1 / z^{2}$ contributions in $\phi_{5}$, which indicates that it likely to be a useful observable in nonperturbative matching calculations for lattice HQET.

Matching conditions for all nineteen parameters have now been investigated [40], a task that has provided considerable insight into the best choice of operators for the final matching procedure. Without ALPT, this simply would not have been feasible in a reasonable timescale.

\subsection{PhySyCAl}

The final package that I would like to discuss is PhySYCAl, which takes a completely different approach to automating LPT. PhySyCAl is a computer algebra system (CAS), i.e. a software package for symbolic manipulation of mathematical expressions, developed by Christoph Lehner [24].

The structure of PhySyCAl is outlined in Figure 4. The heavy lifting is done by three $\mathrm{C}++$ libraries: libcas, the CAS; libqft, a quantum field theory library; and libint, a library for numerical evaluation of algebraic expressions.

The CAS library libcas parses algebraic expressions from text and xml input files and simplifies these expressions. libqft performs all manipulations associated with typical perturbative calculations: deriving vertices from actions and operators; carrying out Wick contractions; and even computing loop integrals in dimensional regularisation via the Passarino-Veltmann reduction. Finally, the loop integrals are evaluated numerically, using a choice of integrators, with libint.

\subsection{Matching relativistic heavy quarks with PhySyCAl}

In the Columbia formulation, used by the RBC/UKQCD Collaboration for its $B$ physics pro- 


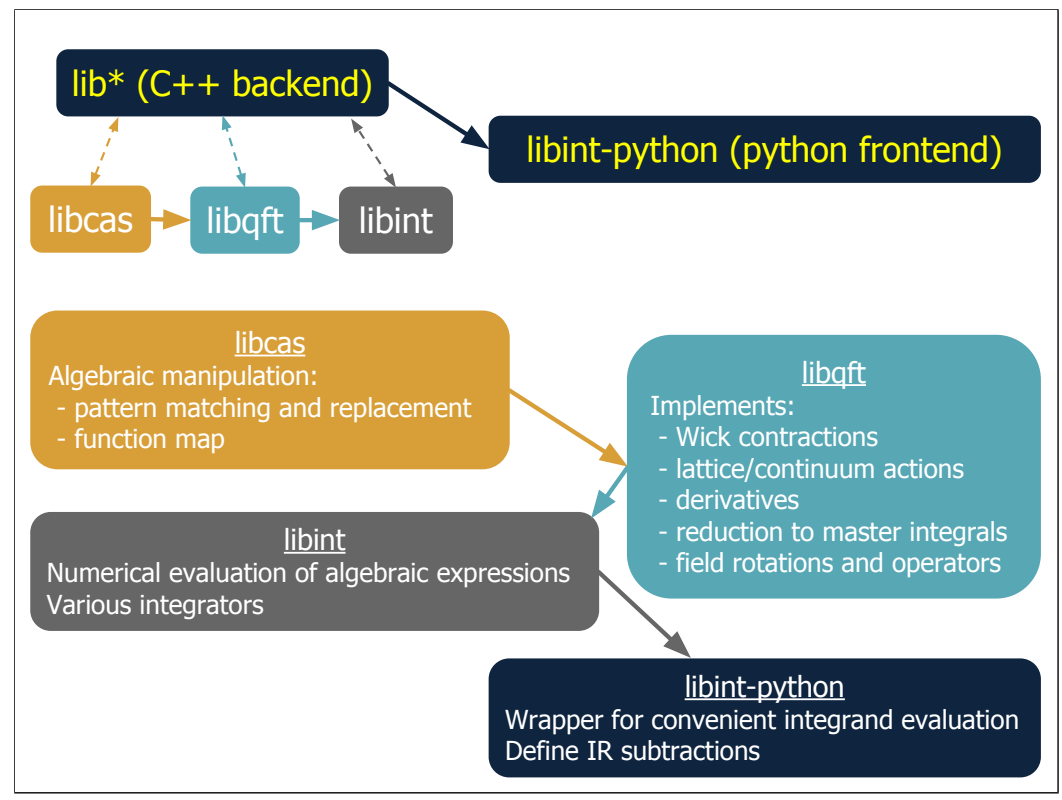

Figure 4: Schematic representation of the structure of PhySyCAl. See accompanying text for details.

gramme, the RHQ action may be written as

$$
\mathscr{S}=\sum_{x} \bar{Q}(x)\left[\left(\gamma_{0} D_{0}-\frac{D_{0}^{2}}{2}\right)+\zeta \sum_{i=1}^{3}\left(\gamma_{i} D_{i}-\frac{D_{i}^{2}}{2}\right)+m_{0}+\frac{i c_{P}}{4} \sum_{\mu, v=0}^{3} \sigma_{\mu v} F_{\mu v}\right] Q(x),
$$

where the parameters $\zeta, m_{0}$, and $c_{P}$ can be tuned to remove discretisation effects [24]. Applying a field rotation to the heavy quark fields, $Q(x) \rightarrow Q^{\prime}(x)=Q(x)+d_{1} \sum_{i=1}^{3} \gamma_{i} D_{i} Q(x)$, the rotated fields may be matched to continuum fields. Since this rotation leaves the mass spectrum unchanged, the three parameters $\zeta, m_{0}$, and $c_{P}$ can be tuned without knowledge of $d_{1}$.

Matching the RHQ quark two-point function to continuum QCD allows one to tune $m_{0}$ via the pole mass and $\zeta$ using the dispersion relation. The parameter $c_{P}$ is extracted by matching the quarkquark-gluon three-point function in the on-shell limit. There are 37 Feynman diagrams required to match $c_{P}$ to one-loop, which is clear evidence that automating the procedure dramatically simplifies the calculation.

PhySyCAlwas used to test the matching framework for the RHQ quarks, by calculating the one-loop coefficients of $\zeta, m_{0}$, and $c_{P}$. Sample data for both $\zeta$ and $c_{P}$ are presented in Figure 5. These perturbative results were compared to the nonperturbatively tuned values, serving as a crosscheck of both methods. It is clear from Figure 5 that perturbative and nonperturbative results agree within errors. Nonperturbative tuning is computationally expensive and currently not available for all operators relevant to the RBC/UKQCD $B$ physics programme. ALPT, implemented using the PhySyCAl package, offers an alternative method for determining renormalisation parameters and tuning the RHQ action that is likely to prove highly useful in the near future. 

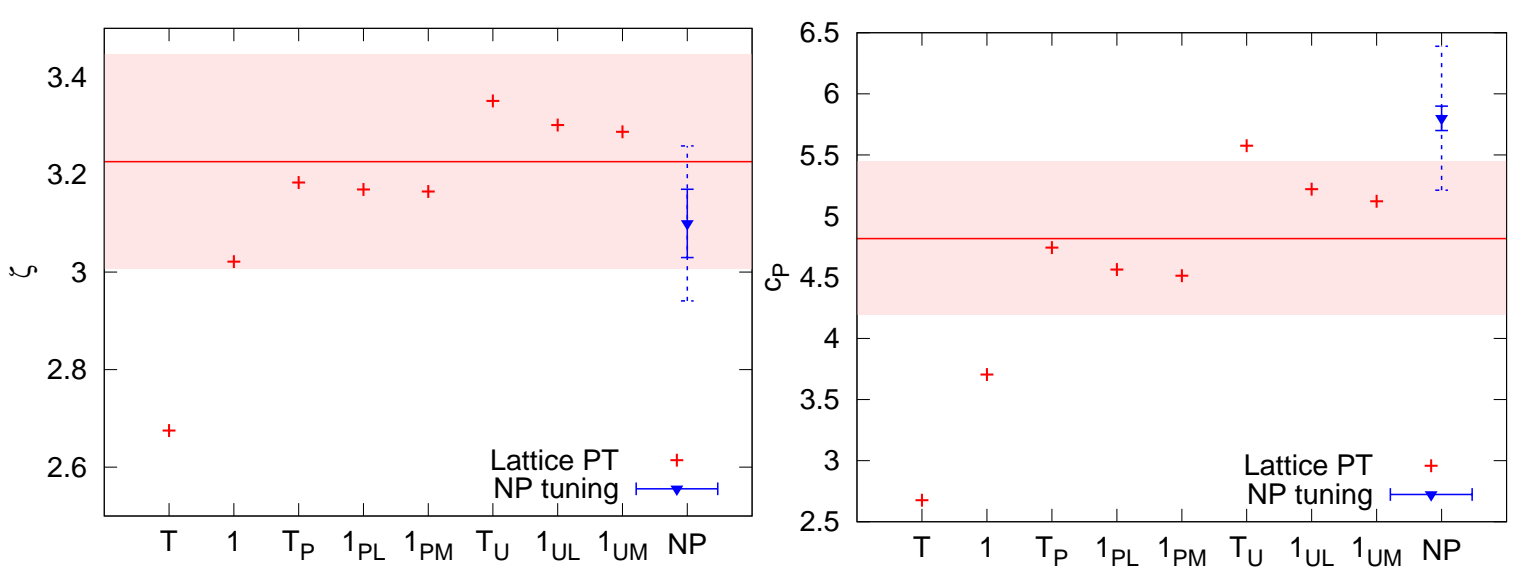

Figure 5: Perturbative and nonperturbative results for the RHQ parameters $\zeta$ (on the left) and $c_{P}$ (on the right). The different points correspond to different choices of perturbative coefficient, improvement scheme and coupling constant. For full details see [24]. The nonperturbative result is plotted in blue for comparison.

\section{Numerical Stochastic Perturbation Theory}

Numerical stochastic perturbation theory (NSPT) lies on a rather different branch of the evolutionary tree of approaches to LPT than the automated routines just discussed. Proposed in [41], NSPT is a numerical application of stochastic quantisation, particularly suited to high-order calculations (for a good review see, for example [42]).

Stochastic quantisation proceeds by introducing an extra degree of freedom, the stochastic time $\tau$, in which the fields evolve according to a Langevin equation with Gaussian noise.

The central claim of stochastic quantisation is that stochastic averages, taken over an ensemble of statistical noise sources $\eta$, converge to the original functional integral expectation values of the theory in the infinite stochastic time limit. That is,

$$
\langle O[\phi(x, \tau)]\rangle_{\eta} \stackrel{\tau \rightarrow \infty}{\longrightarrow}\langle O[\phi(x)]\rangle,
$$

where the stochastic average on the left is over the Gaussian noise and the right hand side is the usual field theoretic expectation value.

Stochastic perturbation theory is obtained by decomposing the action into a free field component and an interacting contribution that is a function of the bare coupling constant. One then iteratively solves the Langevin equation for the full theory as a series in the coupling constant. In the infinite stochastic time limit, the original perturbative expansion is recovered. NSPT is the numerical implementation of this procedure.

In the interests of brevity I have skipped over many interesting subtleties, such as incorporating fermions, stochastic gauge fixing and navigating numerical instabilities, that are beyond the scope of these proceedings and refer the reader to the literature (see, for example, [1]).

\subsection{Recent calculations with NSPT}

There have been a wide range of NSPT calculations carried out since its inception and I discuss here only the most recent results. 


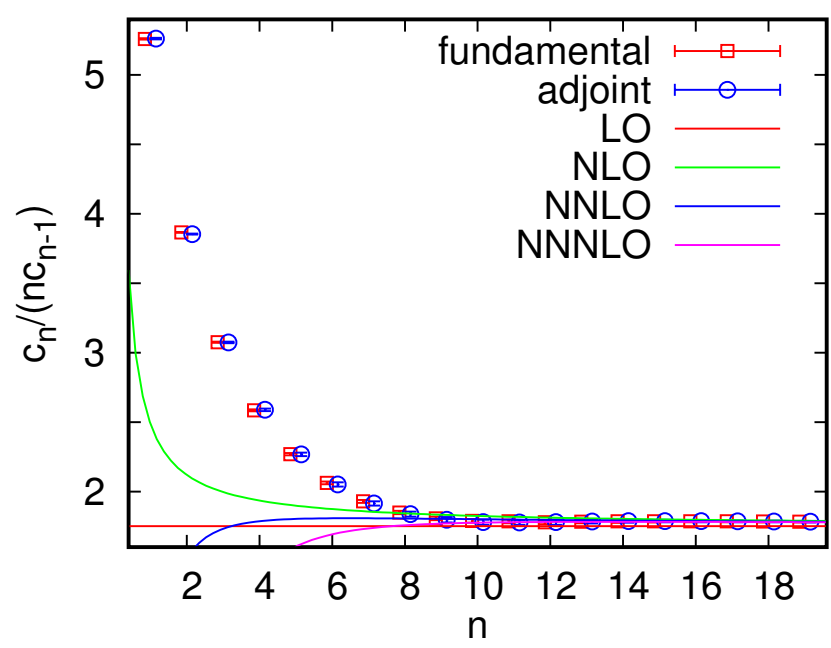

Figure 6: Plot showing the $n$ ! growth of coefficients in the self-energy of a static field source in $S U(3)$ gauge theory. Results for the fundamental representation are shown in red and for the adjoint representation in blue. Plotted lines illustrate the expected behaviour from leading order (LO, in red) up to next-to-next-tonext-to-leading order (NNNLO, in pink) perturbation theory. Plot simplified from [43].

Evidence for renormalons In [43] strong evidence for the existence of renormalons was obtained by studing the Polyakov loop at very high orders, up to $\mathscr{O}\left(\alpha^{20}\right)$. The main results are summarised in Figure 6, where the $n$ ! growth of coefficients characteristic of renormalons is evident. In fact, the normalisation of the leading renormalon of the heavy quark pole mass, which is related to the Polyakov line, was found to differ from zero by ten standard deviations, providing compelling numerical evidence for the existence of the leading infrared renormalon.

Discretisation errors in the MiniMom scheme NSPT has recently been used to investigate and correct discretisation errors in the strong coupling constant, defined in the "Minimal MOM" scheme [44]. The Minimal MOM coupling is renormalisation-scale invariant, but on the lattice this invariance is slightly broken by lattice artefacts at $\mathscr{O}\left(a^{2}\right)$. These effects are seen in the plot on the lefthand side of Figure 7, where the points do not lie perfectly on top of each other. These artefacts are mainly due to loop contributions to the ghost and gluon propagators used to extract the coupling. Such discretisation effects become more important for unquenched lattice calculations, where very fine lattices are prohibitively expensive.

Previous attempts to calculate the discretisation corrections at one loop in standard LPT proved insufficient to remove the lattice artefacts completely [45] and higher orders were required. The righthand side of Figure 7 shows the effect of the three-loop corrections calculated with NSPT: the points at different spatial momenta now lie almost on top of each other. Although only a preliminary study in the quenched approximation, these results demonstrate the effectiveness of NSPT as a tool for correcting lattice artefacts.

NSPT and the gradient flow The gradient flow is a technique similar in spirit to stochastic quantisation, but without the inclusion of a stochastic noise term in the evolution equation. The evolution in the flow time, $\tau$, is governed by a deterministic partial differential equation that drives 


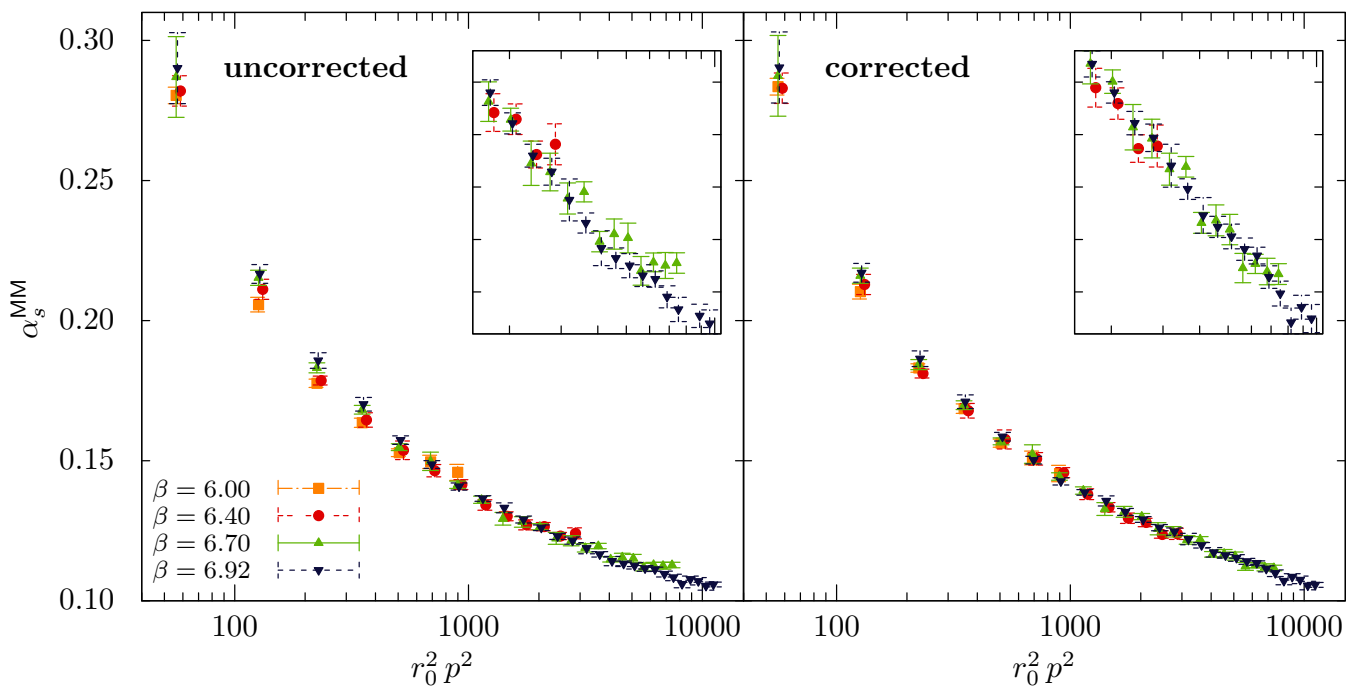

Figure 7: Coupling constant in the "Minimal MOM" scheme [44] for quenched QCD at fixed physical volume, before (left) and after (right) correcting for discretisation effects [46]. The corrections were calculated using NSPT at three loops and results are plotted at four different values of $\beta$.

the fields to a stationary point of the action, a process that corresponds to repeatedly smearing the fields. For a review of new developments involving the gradient flow, see [47].

NSPT has recently been applied to the gradient flow method in the Schrödinger functional scheme [48]. In particular, the gauge field strength, $\mathscr{E}$, was studied and the results of [49] for the normalisation of $\mathscr{E}$ were reproduced. The use of NSPT allowed this result to be extended to the next two orders. Figure 8 shows the field strength at two-loops, $\mathscr{E}(2)$, as function of the stochastic step size, $\varepsilon$, and for five choices of flow time constant $c$. This constant relates the flow time, $\tau$, to the box size, $L$, via $c^{2}=8 \tau / L^{2}$ and different choices represent different renormalisation schemes for the finite volume coupling constant defined through $\mathscr{E}$ [49]. The final errors in the two-loop field strength are only $3 \%$, suggesting that NSPT will be a promising tool for precision calculations in the Schrödinger functional scheme in the future (see also [50]).

\section{Summary}

As lattice calculations become more precise, the need to reduce all sources of systematic uncertainty grows more pressing. LPT plays an important role in reducing systematic uncertainties, from improving actions to calculating renormalisation parameters and mixing coefficients. As lattice actions become more sophisticated, automation becomes a necessity and the effectiveness of ALPT has been demonstrated in a wide variety of calculations over the last ten years. Even in theories requiring nonperturbative renormalisation, ALPT has a role to play as a guide to choosing suitable renormalisation conditions and as a test of nonperturbative results.

In many calculations perturbative uncertainties dominate the error budgets and improved and higher-order calculations are required. ALPT, perhaps combined with weak coupling calculations, 


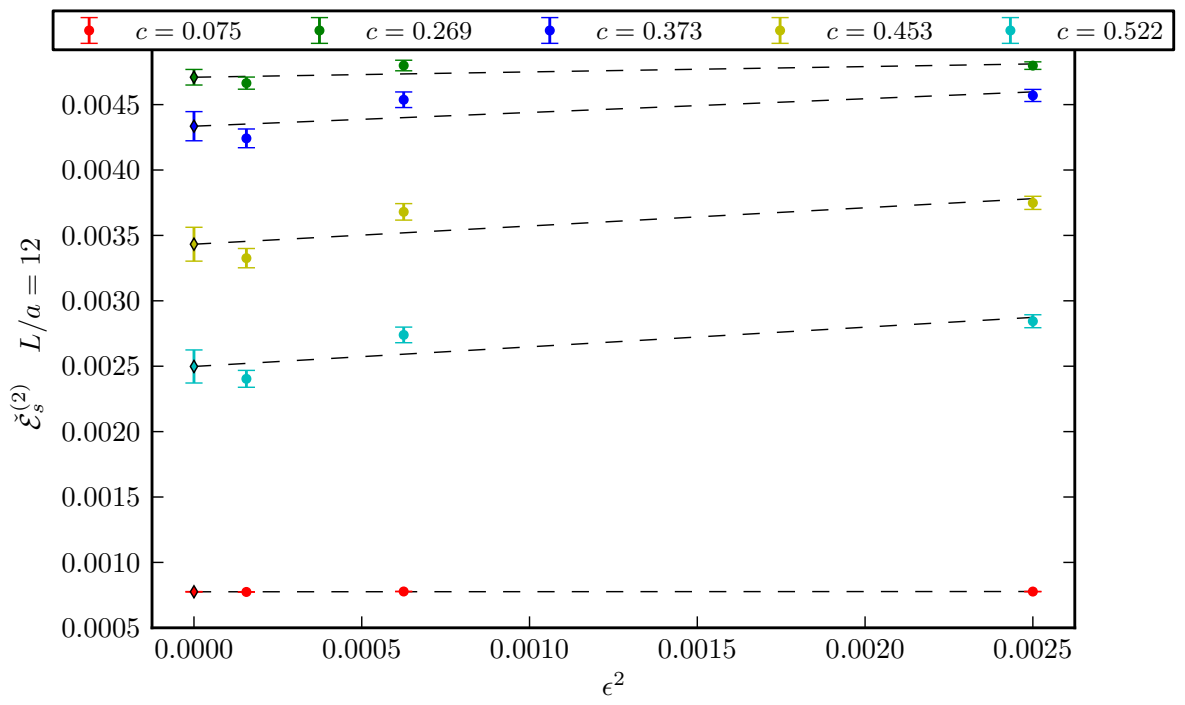

Figure 8: A plot of the two-loop field strenth, $\mathscr{E}^{(2)}$, against stochastic time step size. Each choice of $c$, where $c^{2}=8 \tau / L^{2}$ (see accompanying text), represents a different renormalisation scheme.

and NSPT will certainly be the only realistic methods for carrying out higher-order calculations in the future. With the advent of new automation routines, the use of ALPT is only set to grow.

\section{Acknowledgments}

I would like to thank Christoph Lehner, Dirk Hesse and Piotr Korcyl for many informative conversations about ALPT and all the authors who contributed their time and their plots for this plenary. Particular thanks to Chris Bouchard and Kelcie Ralph for reading a draft of the manuscript.

\section{References}

[1] For an extensive list of LPT references, see latticeperturbationtheory.org.

[2] Y. Aoki et al., (2012), 1206.2554

[3] M. Lüscher et al., Nucl.Phys.B 478 (1996) 365, [hep-lat/ 9605038 ]

[4] M. Lüscher, P. Weisz and U. Wolff, Nucl.Phys.B 359 (1991) 221

[5] G. Martinelli et al., Nucl.Phys.B 445 (1995) 81, [hep-lat/9411010]

[6] H.-W. Lin and N.H. Christ, Phys.Rev.D 76 (2007) 074506, [hep-lat/ 0608005 ]

[7] K. Orginos, these proceedings

[8] G.P. Lepage et al., Nucl.Phys.(Proc.Suppl.) 83, 866 (2000), [hep-lat/ 9910018 ]

[9] F. Di Renzo and L. Scorzato, JHEP 10, 038 (2001), [hep-lat/ 0011067 ]

[10] R. Horsley, P.E.L. Rakow and G. Scheierholz, Nucl.Phys.(Proc.Suppl.) 106, 870 (2002)

[11] H.D. Trottier et al., Phys.Rev.D 65 (2002) 094502 [hep-lat/ 0111028 ]

[12] K. Wong, H. Trottier and R. Woloshyn, Phys.Rev.D 73 (2006) 094512, [hep-lat/ 0512012 ]

[13] I.F. Allison et al., (2008), PoS (Latti ce2008) 225

[14] G.P. Lepage and P.B. Mackenzie, Phys.Rev.D (1993) 482250 
[15] S.J. Brodsky, G.P. Lepage and P.B. Mackenzie, Phys.Rev.D 28 (1983) 228

[16] K. Hornbostel, G.P. Lepage and C. Morningstar, Phys.Rev.D 67 (2002) 034023

[17] M. Constantinou, H. Panagopoulos and A. Skouroupathis, PoS (Latt i ce2006) 155; H. Panagoupoulos and E. Vicari, Phys.Rev.D 59 (1999) 057503 [hep-lat/ 9809007 ]

[18] M. Lüscher and P. Weisz, Nucl.Phys.B 266 (1986) 309

[19] B. Allés et al., Nucl.Phys.B 413 (1994) 553, [hep-lat/9301012]

[20] S. Capitani and G. Rossi, (1995), hep-lat/9504014

[21] A. Hart et al., J.Comput.Phys. 209 (2005) 340, [hep-lat/ 041102 6]; A. Hart et al., Comput.Phys.Commun. 180 (2009) 2698, [0 904 . 0375]

[22] P. Ribeca, Nucl.Phys.B(Proc.Suppl.) 140 (2005) 731; S. Takeda, Nucl.Phys.B 811 (2009) 36, [0808.3065]

[23] D. Hesse, R. Sommer and G.M. von Hippel, PoS (Lattice2011) 20 9; D. Hesse and R. Sommer, (2012), 1211.0866; D. Hesse, (2012), PoS (Lattice2012) 234

[24] C. Lehner, (2012), PoS (Lattice2012) 126; see PhySyCAl webpage www.lhnr.de/physyhcal/

[25] G.P. Lepage, (1980), CLNS-80/447

[26] C. Morningstar, Phys.Rev.D 48 (1993) 2205, [hep-lat/9301005]

[27] G.M. von Hippel, Comput. Phys. Commun. 181 (2010) 705, [0910 . 5111]

[28] A. Hart, R.R. Horgan and L.C. Storoni, Phys.Rev.D 70 (2004) 034501, [hep-lat / 0402033 ]; A. Hart, G.M. von Hippel and R.R. Horgan, Phys.Rev.D 75, (2007) 014008, [hep-lat / 0605007 ]; E.H. Müller, A. Hart and R.R. Horgan, Phys.Rev.D 83 (2011) 034501, [1011. 1215]

[29] R.J. Dowdall et al., Phys.Rev.D 85 (2012) 054509, [1110 . 6887]

[30] C.J. Monahan, Mod. Phys. Lett. A, 27 (2012) 1230040, [1210. 7266 ]

[31] T.C. Hammant et al., Phys. Rev. Lett. 107 (2011) 112002, [1105. 530 9]; T.C. Hammant et al., Phys.Rev.D. 88 (2013) 014505, [1303 .3234]

[32] C. McNeile et al., Phys.Rev.D 82 (2010) 034512 [1 004.428 5]; C. McNeile et al., Phys.Rev.D 85 (2012) 031503 [1110.4510]; C. McNeile et al., Phys.Rev.D 86 (2012) 074503 [1207. 0994 ];

[33] R. Dowdall et al., (2013), [1309.5797]

[34] J. Beringer et al., (Particle Data Group), Phys.Rev.D 86 (2012) 010001, online updates at pdf.lbl.gov

[35] A. Lee et al., Phys.Rev.D 87 (2013) 074018, [1302 .3739]

[36] K. Melnikov and T. van Ritbergen, Phys. Lett. B 482 (2000) 99, [hep-ph/9912391]

[37] A. Gray et al., Phys.Rev.D 72 (2005) 094507, [hep-lat/ 0507013 ]

[38] D. Hesse, Automated lattice perturbation theory in the Schrödinger Functional, (2012), Ph.D. thesis, Humboldt-Universität zu Berlin

[39] R. Sommer, (2010), 1008.0710

[40] P. Korcyl, PoS (Beauty2013) 071; P. Korcyl, these proceedings

[41] F. Di Renzo et al., Nucl.Phys.B (Proc. Suppl.) 34 (1994) 795; F. Di Renzo et al., Nucl.Phys.B 426 (1994) 675 [hep-lat/9405019]

[42] F. Di Renzo and L. Scorzato, JHEP 0410 (2004) 073 [hep-lat / 0410010 ]

[43] G. Bali et al., Phys.Rev.D 87 (2013) 094517 [1303.3279]; C. Bauer, G.S. Bali and A. Pineda, Phys.Rev.Lett. 108 (2012) 242002 [1111.3946]; A. Pineda, these proceedings

[44] L. von Smekal, K. Maltman and A. Sternbeck, Phys.Lett.B 681 (2009) 336, [hep-ph/ 0903.1696 ]

[45] A. Sternbeck et al., PoS (Lattice 2012) 243

[46] J. Simeth, these proceedings

[47] M. Lüscher, (2013), 1308.5598

[48] M. Dalla Brida, these proceedings

[49] P. Fritzsch and A. Ramos, JHEP 1310 (2013) 008, [1301 . 4388]

[50] M. Brambilla, these proceedings 